Provided for non-commercial research and educational use only. Not for reproduction or distribution or commercial use.

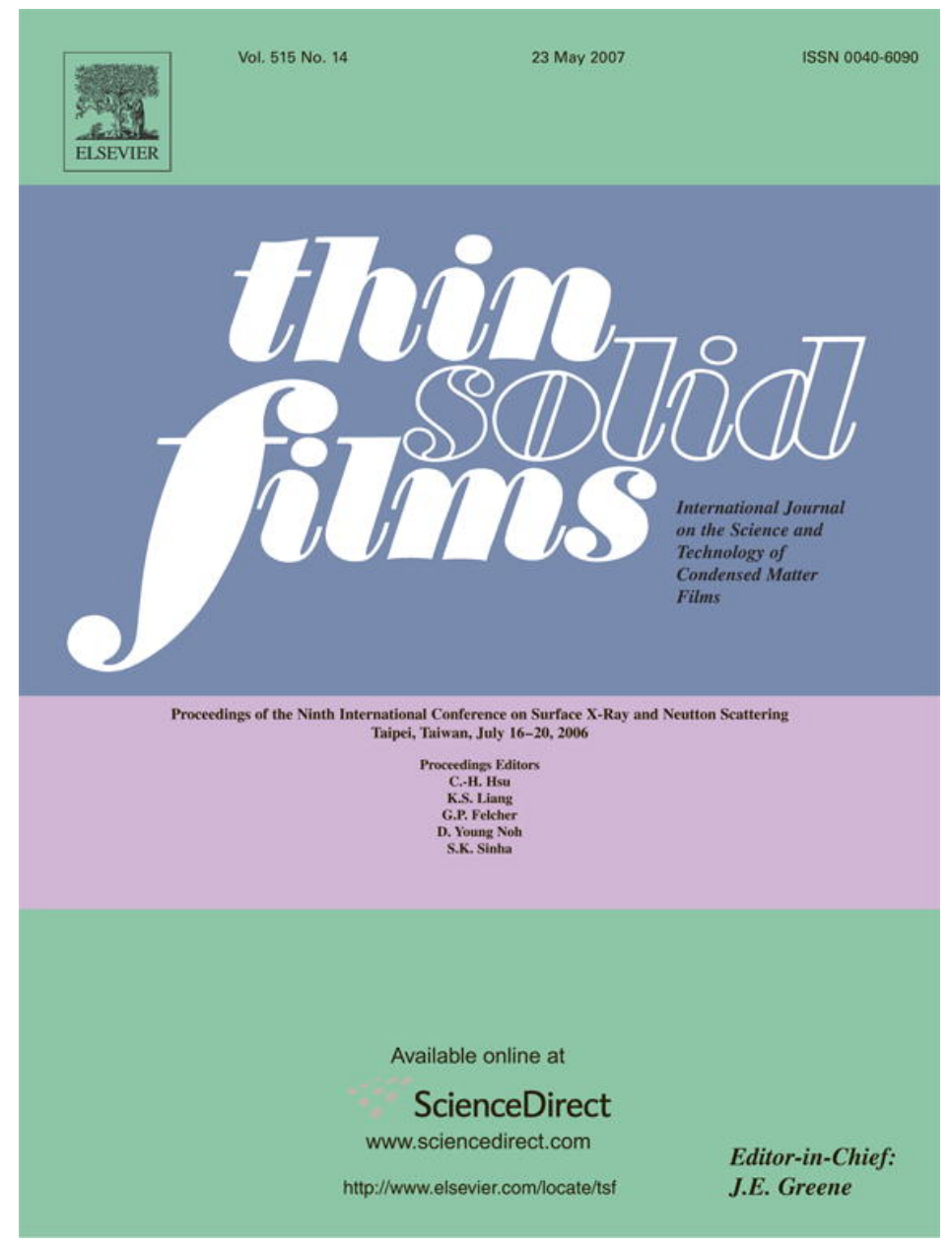

This article was originally published in a journal published by Elsevier, and the attached copy is provided by Elsevier for the author's benefit and for the benefit of the author's institution, for non-commercial research and educational use including without limitation use in instruction at your institution, sending it to specific colleagues that you know, and providing a copy to your institution's administrator.

All other uses, reproduction and distribution, including without limitation commercial reprints, selling or licensing copies or access,

or posting on open internet sites, your personal or institution's website or repository, are prohibited. For exceptions, permission may be sought for such use through Elsevier's permissions site at: 


\title{
Coherent X-ray imaging of individual islands in GISAXS geometry
}

\author{
I.A. Vartanyants ${ }^{\mathrm{a}, *}$, D. Grigoriev ${ }^{\mathrm{b}}$, A.V. Zozulya ${ }^{\mathrm{a}}$ \\ ${ }^{\text {a } H A S Y L A B, D E S Y, ~ N o t k e s t r . ~ 85, ~ H a m b u r g ~ D-22607, ~ G e r m a n y ~}$ \\ ${ }^{\mathrm{b}}$ Insitut für Physik, Humboldt-Universität zu Berlin, Newtonstr. 15, 12489, Berlin, Germany
}

Available online 22 January 2007

\begin{abstract}
In this paper we give theoretical analysis of an image formation and reconstruction of individual islands of nano-size dimensions in the coherent GISAXS experiments. It is shown that there are some favorable conditions with incident angle close to the critical angle when GISAXS diffraction patterns originating from the individual islands can be directly inverted using iterative phase retrieval techniques.
\end{abstract}

(C) 2006 Elsevier B.V. All rights reserved.

Keywords: Coherent X-ray diffraction; Phase retrieval; Quantum dots

\section{Introduction}

Coherent X-ray Diffraction (CXD) is a new and fast developing imaging technique in scattering. The method is based on the following principles: if an object of finite size is coherently illuminated and its diffraction pattern is sampled finer than at the twice the frequency required by Shannon's theorem then it can be uniquely inverted to give a real space image of the sample. This basic idea leads to a development of a lensless microscope, whose objective lenses are substituted by powerful inversion algorithms, which can, in principle, lead to the development of a high resolution and aberration free imaging technique.

The basic ideas of the method were first developed in visible light optics [1,2] and crystallography [3], where it was called an oversampling method. After first successful demonstration for $1 \mathrm{D}$ and $2 \mathrm{D}$ objects [4-6] this diffractive imaging approach was used in a number of coherent X-ray scattering experiments on test objects for $2 \mathrm{D}$ and $3 \mathrm{D}$ imaging of inorganic and even organic samples [7-12]. In these CXD experiments on noncrystallographic objects the forward scattering geometry was used. If a crystalline sample is used then the whole reciprocal space can be effectively measured with coherent X-ray beams by the angular (or energy) scan near its Bragg peaks. Applying the phase retrieval methods allow the shape and the internal

\footnotetext{
* Corresponding author. Tel.: +49 0408998 2653; fax: +49 04089984475.

E-mail address: Ivan.Vartaniants@desy.de (I.A. Vartanyants).
}

structure of the crystalline particle to be obtained with a model independent approach $[13,14]$. If strain is present in the crystalline particle this will effectively lead to an additional phase factor in the real space image of the particle [15] and, as it was shown in the recent experiment, can be also uniquely retreived from the CXD measurements [16]. In the future, with the availability of the 4-th generation X-ray sources in the form of Free Electron Lasers (FEL) [17,18] that will be nearly fully coherent and have a unique femtosecond time structure this approach can be even used for single molecule imaging [19].

The first CXD experiments were performed on a specially prepared or artificially fabricated samples necessary for testing the method. But it is of great practical and scientific interest to apply this approach to classes of samples that are currently most important in the modern nano-physics and nano-technology. In this regard, a good candidate for CXD imaging could be quantum wires and quantum dots that are presently on the cutting edge of research due to their wide applications in the optoelectronics and semiconductor industry [20]. It has been shown in many studies that the electronic properties of nanodevices based on quantum structures are first of all determined by their shape, which is a strong function of the growth conditions.

Different X-ray scattering and diffraction techniques are successfully used in study of the average shapes, sizes, correlation lengths, composition and strain of the quantum structures. The most effective are grazing incidence techniques, when penetration depth of the incoming X-ray beam can be 
varied and surface structures can be effectively studied. These are grazing incidence diffraction (GID) and grazing incidence small angle X-ray scattering (GISAXS). A detailed description of conventional X-ray scattering techniques and novel methods (such as GID and GISAXS) developed for the study of quantum structures can be found in the books $[21,22]$. What is common for all these X-ray scattering methods is that the structural information (shape, size and deformation) can be obtained only indirectly by comparing results of the experiment with simulated diffraction pattern obtained from realistic modeling. In this paper we propose to use CXD as a new method for high resolution imaging of quantum dots with a model independent approach.

The problem of imaging of quantum dot structures with coherent X-rays lies in a dramatically reduced scattered signal when the size of the particles goes below $100 \mathrm{~nm}$. In the previous papers $[23,24]$ the possibility of CXD imaging experiments on a periodic array of quantum dots was analyzed theoretically and experimentally. It was shown that the use of periodic arrays can enhance the scattering signal and make measurements with a 3-rd generation synchrotron sources feasible. It was also demonstrated that diffraction pattern from such a periodic array of quantum dots can be inverted and can produce an averaged shape of an island with ten nanometer resolution [24].

In this paper we want to analyze the special situation of coherent scattering from a single island of nanoscale dimensions measured in a GISAXS geometry. Though this situation looks purely academic at the moment we think that it could be experimentally studied in the nearest future. The possibility of CXD experiments on particles of few hundred nanometer size was recently demonstrated [25] when focusing devices in the form of Kirkpatrick-Baez (KB) mirrors or lenses are used at 3rd generation synchrotron sources. Using highly focusing lenses $[26,27]$ with the focus size just about the size of a nanoparticle it will become not only possible to get a strong coherently scattered signal but also to map the position of a particle on a surface. First test experiments with bigger particle sizes and more decent focusing conditions showed the feasibility of this approach [28]. In the future, when X-ray FEL's become available, coherent X-ray scattering experiments on islands of few tens of nanometers size will become possible [17].

Reconstruction algorithms used in CXD imaging experiments are based on the fact that the kinematical scattering approach can be used. In this case the scattered amplitude is just a Fourier transform of the electron density of the sample. This approach is justified by small sizes of objects and a weak X-ray scattering in the sample, when multiple scattering effects can be neglected. At the same time, it is well known [29] that in the grazing incidence scattering geometry due to a small incoming angles the 1-st Born approximation (or kinematical scattering) fails to describe correctly the scattering process and instead the Distorted Wave Born Approximation (DWBA) has to be used [30]. ${ }^{1}$ Total amplitude in DWBA is a coherent sum of several

\footnotetext{
${ }^{1}$ Theory of the DWBA scattering in GISAXS geometry on the supported islands was developed in [31]. See also for an overview [32].
}

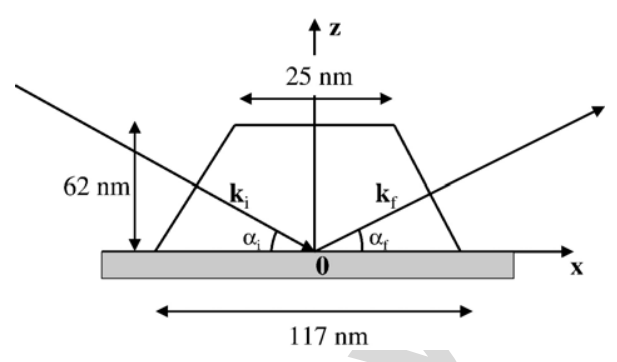

Fig. 1. Schematic view of a scattering process on a single Ge island grown on a Si substrate. The shape and dimensions of an island correspond to that used in GISAXS calculations.

scattered amplitudes. It also means that there is no simple relationship between the scattered amplitude and the electron density of the sample in the form of a single Fourier transform. In this situation iterative phase retrieval algorithm that is based on a multiple use of the Fourier transform back and forth between real and reciprocal space, in principle, cannot be used directly for reconstruction. The purpose of our research was to analyze the scattering process in details and to find situations when reconstruction from coherent GISAXS experimental data can still give adequate results.

The paper is organized as follows. In Section 2 a short overview of the DWBA scattering theory will be given. In Section 3 results of theoretical calculations of coherent diffraction patterns in GISAXS geometry for a specific shape of an island will be discussed. In Section 4 we present results of reconstruction of a single island obtained with different scattering conditions and in the final section we give a summary of our results.

\section{Theory of DWBA scattering}

In the frame of the DWBA theory the transition amplitude between the initial state described by the wave vector $\mathbf{k}_{i}$ and the final state described by the wave vector $\mathbf{k}_{f}$ can be written as [33]

$A_{\text {tot }}\left(\mathbf{k}_{i}, \mathbf{k}_{f}\right)=\left(\frac{\mathbf{k}^{2}}{4 \pi}\right) \int_{V} E\left(\mathbf{r},-\mathbf{k}_{f}\right) \delta \chi(\mathbf{r}) E\left(\mathbf{r}, \mathbf{k}_{i}\right) d \mathbf{r}$.

This general result can be also obtained as a consequence of a reciprocity theorem in optics [34]. In Eq. (1) $E\left(\mathbf{r}, \mathbf{k}_{i}\right)$ and $E\left(\mathbf{r},-\mathbf{k}_{f}\right)$ are the scalar ${ }^{2}$ wavefields corresponding to undisturbed solutions of the scattering problem and $\delta \chi(\mathbf{r})$ is the perturbation of the susceptibility. Exact solutions for the scattering of the plane wave by the smooth surface are given by Fresnel theory [34]

$E(\mathbf{r}, \mathbf{k})=\left\{\begin{array}{c}e^{i \mathbf{k r}}+R(\mathbf{k}) e^{i \mathbf{k}_{r} \mathbf{r}}, z>0 \\ T(\mathbf{k}) e^{i \mathbf{k}_{t} \mathbf{r}}, z<0,\end{array}\right.$

where $\mathbf{k}_{r}$ and $\mathbf{k}_{t}$ are the wave vectors of the specularly reflected and transmitted beams and $R(\mathbf{k})$ and $T(\mathbf{k})$ are Fresnel reflection and transmission coefficients respectively.

\footnotetext{
${ }^{2}$ Here we will consider only one component of the vector wavefield.
} 
In the following we will consider a simple situation of scattering on a single island with a constant composition that will be considered as a perturbation in DWBA theory. In this case variation of the susceptibility $\delta \chi(\mathbf{r})$ can be directly related to the shape function $s(\mathbf{r})$ of an island as $\delta \chi(\mathbf{r})=\chi_{0} s(\mathbf{r})$, where $\chi_{0}$ is the zero-order Fourier component of the susceptibility. The shape function is determined in a usual way being zero outside of an island and one inside.

In the case of GISAXS scattering geometry and our choice of the coordinate system (Fig. 1) only the amplitudes of the incident and specularly reflected waves have to be considered for scattering from an island. Substituting expressions of the initial and final states of the wavefields in Eq. (2) at $z>0$ into Eq. (1) we immediately get an expression for the transition amplitude as a coherent sum of four scattering events

$A_{\text {tot }}\left(\mathbf{k}_{i}, \mathbf{k}_{f}\right)=A_{1}+A_{2}+A_{3}+A_{4}$,

where

$A_{1}(\mathbf{q})=\chi_{0}\left(\frac{\mathbf{k}^{2}}{4 \pi}\right) \int s(\mathbf{r}) e^{-i\left(\mathbf{q}_{\|}+\mathrm{q}_{1}^{z}\right) \cdot \mathbf{r}} d \mathbf{r}$

$A_{2}\left(\mathbf{q}_{\|}, \alpha_{i}\right)=\chi 0\left(\frac{\mathbf{k}^{2}}{4 \pi}\right) R\left(\alpha_{i}\right) \int s(\mathbf{r}) e^{-i\left(\mathbf{q}_{\|}+\mathbf{q}_{2}^{z}\right) \cdot \mathbf{r}} d \mathbf{r}$

$A_{3}\left(\mathbf{q}_{\|}, \alpha_{f}\right)=\chi_{0}\left(\frac{\mathbf{k}^{2}}{4 \pi}\right) R\left(\alpha_{f}\right) \int s(\mathbf{r}) e^{-i\left(\mathbf{q}_{\|}+\mathbf{q}_{3}^{z}\right) \cdot \mathbf{r}} d \mathbf{r}$,

$A_{4}\left(\mathbf{q}_{\|}, \alpha_{i}, \alpha_{f}\right)=\chi_{0}\left(\frac{\mathbf{k}^{2}}{4 \pi}\right) R\left(\alpha_{i}\right) R\left(\alpha_{f}\right) \int s(\mathbf{r}) e^{-i\left(\mathbf{q}_{\|}+\mathrm{q}_{4}^{z}\right) \cdot \mathbf{r}} d \mathbf{r}$

and integration in Eqs. (4)-(7) is performed over the whole space. In expressions (4)-(7) angles $\alpha_{i}$ and $\alpha_{f}$ are the incident and exit angles (Fig. 1). As it follows from these equations there are four scattering channels that are contributing to the total amplitude (Fig. 2): (1) kinematical scattering from the island, (2) specular reflection from the surface followed by kinematical scattering from the island, (3) kinematical scattering from the island followed by specular reflection from the surface, (4)
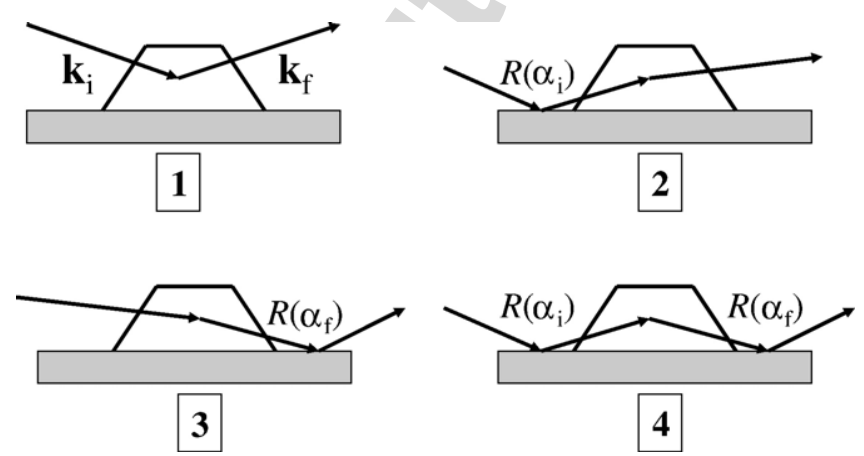

Fig. 2. Four scattering channels giving the main contribution to the total amplitude in the GISAXS geometry. specular reflection from the surface followed by kinematical scattering from the island followed by the specular reflection from the surface.

In Eqs. (4)-(7) the magnitude of the $z$-component of the momentum transfer vector is different for each amplitude and is determined by the following expressions: $\mathrm{q}_{1}^{z}=\mathrm{k}_{f}^{z}-\mathrm{k}_{i}^{z}, \mathrm{q}_{2}^{z}=\mathrm{k}_{f}^{z}+$ $\mathrm{k}_{i}^{z}, \mathrm{q}_{3}^{z}=-\left(\mathrm{k}_{f}^{z}+\mathrm{k}_{i}^{z}\right), \mathrm{q}_{4}^{z}=-\left(\mathrm{k}_{f}^{z}-\mathrm{k}_{i}^{z}\right)$.

Finally, we get for the total intensity describing the process in Eq. (1) the following expression

$I_{\text {tot }}\left(\mathbf{k}_{i}, \mathbf{k}_{f}\right)=\left|A_{\text {tot }}\left(\mathbf{k}_{i}, \mathbf{k}_{f}\right)\right|^{2}=\left|A_{1}+A_{2}+A_{3}+A_{4}\right|^{2}$.

It contains contribution from each channel of scattering as well as interference terms. Eq. (8) is valid only if the whole island is coherently illuminated. In the case of reduced coherence, partial coherence effects should be taken into account [15]. In the case of many islands on the surface illuminated by partially coherent beam expression (8) should be averaged over the islands position and shape distribution (see for e.g. [32]).

\section{GISAXS diffraction patterns from an isolated island}

As a typical sample for GISAXS simulations, we chose a $\mathrm{Ge}$ island grown on a Si surface. An island with the size from $60 \mathrm{~nm}$ to $120 \mathrm{~nm}$ was taken in the form of truncated rectangular pyramid (Fig. 1)). This shape is typical for the selforganized growth and was observed in a number of experiments (see for e.g. [35]).

GISAXS diffraction patterns from this island were calculated according to Eqs. (3)-(8) for different incidence angles in the range from $\alpha_{i}=0.1^{\circ}$ to $\alpha_{i}=0.3^{\circ}$. The incident energy was chosen to be $E_{\text {in }}=8.92 \mathrm{keV}$. Incoming angles below the critical angle of a Si substrate $\alpha_{c}=0.2^{\circ}$ and above the critical angle are covered by these calculations. The value of the exit angle $\alpha_{f}$ was varied from $\alpha_{f}=0$ that corresponds to direction along the surface up to larger exit angles. GISAXS diffraction patterns for incidence angles $\alpha_{i}=0.1,0.2$ and $0.3^{\circ}$ are shown in Fig. 3 . These diffraction patterns are the result of a complicated interference between four scattering channels described in the previous section. Kinematically scattered intensity $I_{\text {kin }}(\mathbf{q})=$ $\left|A_{1}(\mathbf{q})\right|^{2}$ calculated for the perpendicular momentum transfer vector $\mathbf{q}_{z}$ starting from $\mathbf{q}_{z}=0$ is also shown in Fig. 3a for comparison. It contains features expected for an island of the shape depicted in Fig. 1. These are, first of all, strong flares going in three directions perpendicular to three facets of the island. It is nothing else than crystal truncation rods [36] typical for a scattering from a small facetted crystal [15]. Calculations performed at different incidence angles (Fig. 3b,c,d) shows how this initial diffraction pattern is changed due to additional channels of scattering. It is well seen from these figures that diffraction pattern from the same island evolves from the kinematical one depending on the incidence angle. At angles lower than the critical angle the broadening of the side flares can be observed. For larger incidence angles these flares finally split into two flares. For incidence angles higher than critical a strong flares below the specular angle can be observed. 
(a)
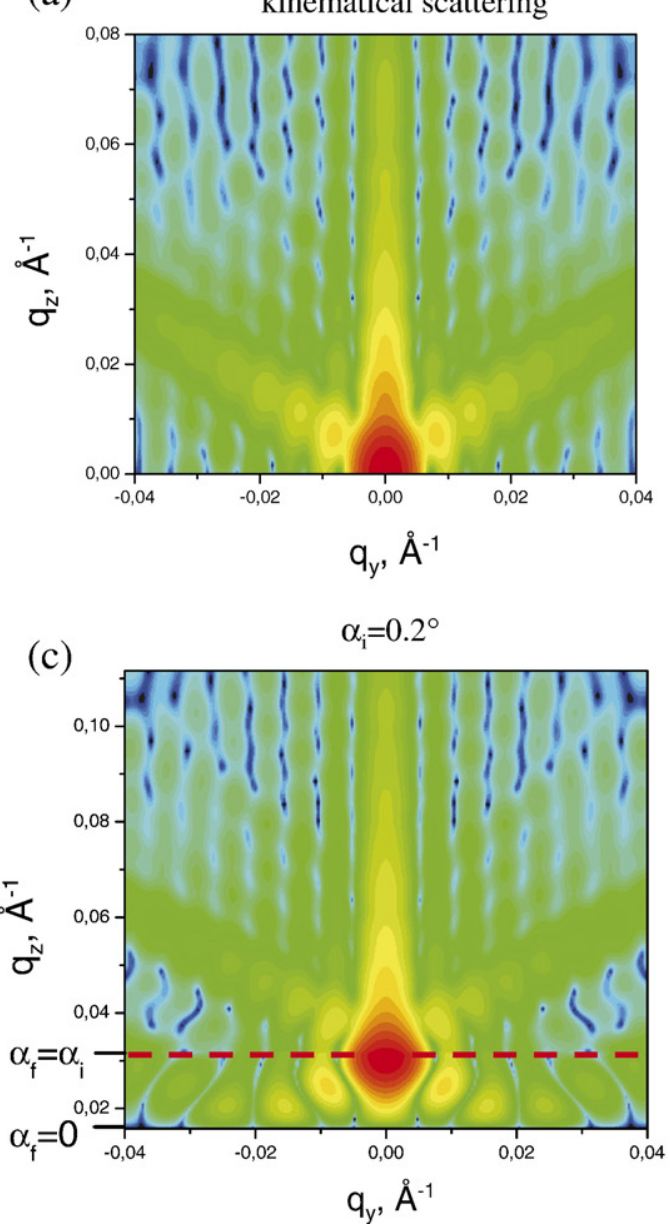

(b)
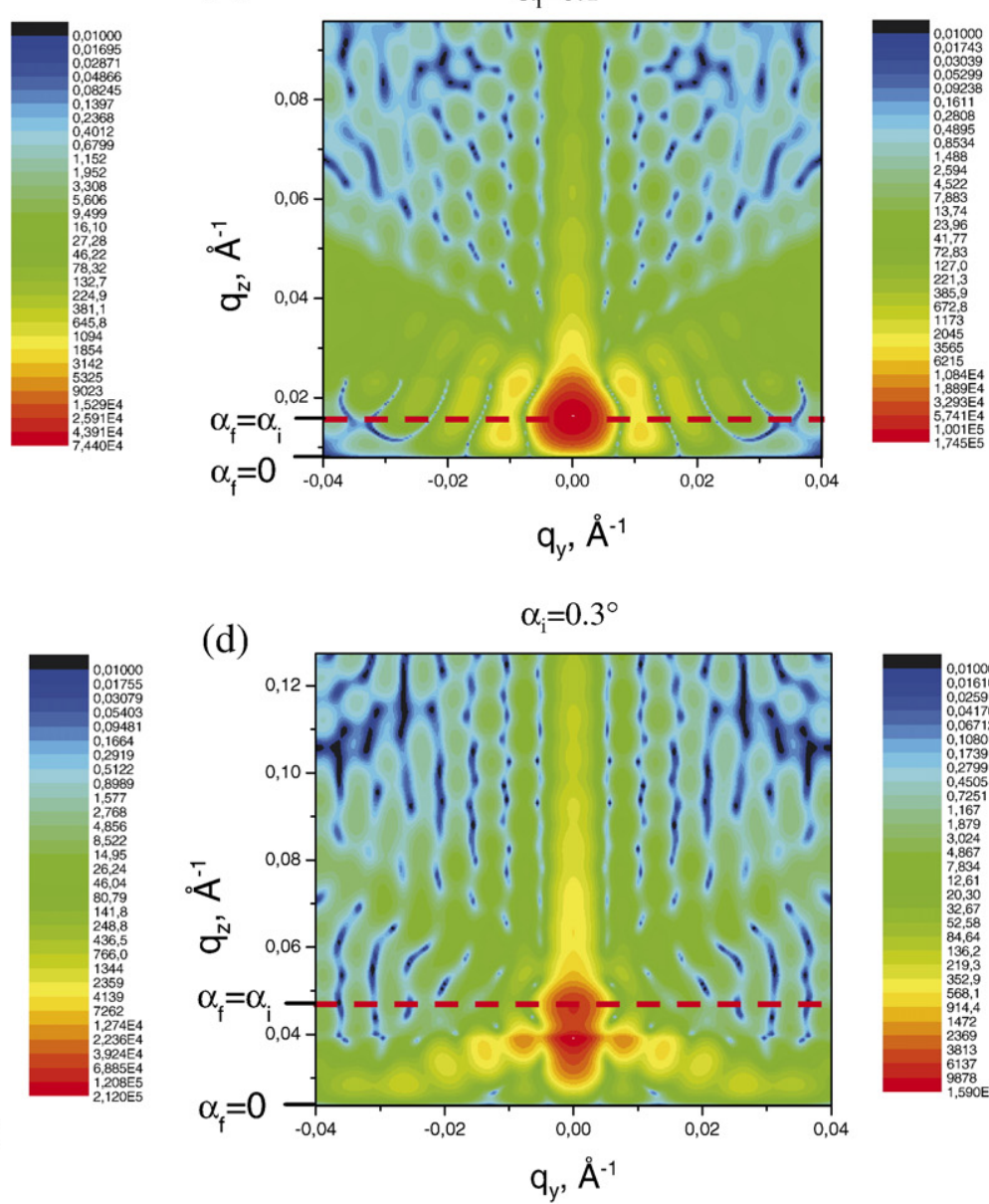

(d)

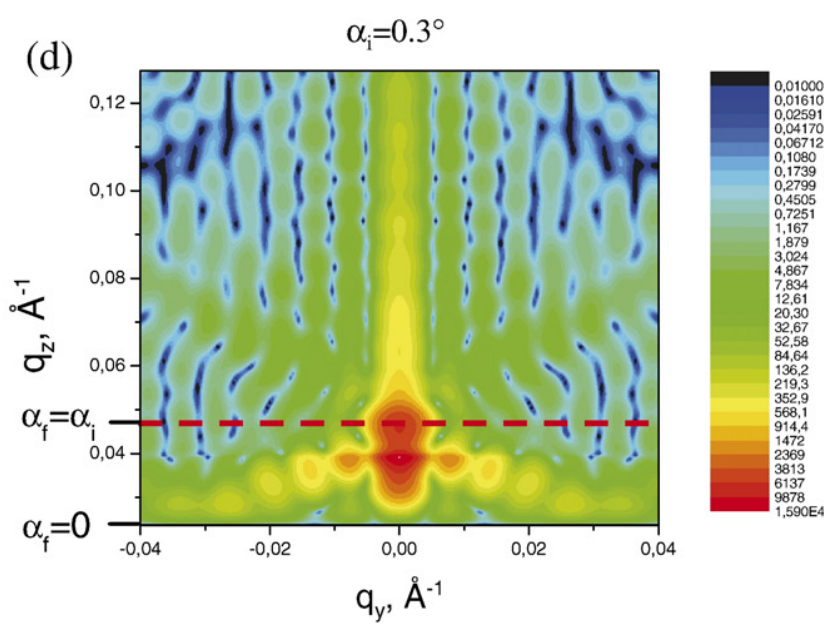

Fig. 3. GISAXS diffraction patterns calculated for different incidence angles. For comparison kinematically scattered intensity is also shown. For each incidence angle part of the diffraction pattern from $\alpha_{f}=\alpha_{i}$ (indicated by dash line) to higher values of the exit angle was used in reconstruction of an island shape.

More careful examination of GISAXS diffraction patterns has revealed an interesting fact. Diffraction patterns calculated for the incidence angle equal to the critical angle and for the exit angles starting from the specular $\left(\alpha_{f}=\alpha_{i}\right)$ to higher values remarkably resembles the features of kinematically scattered intensity $I_{\text {kin }}(\mathbf{q})$ (compare Fig. 3a,c). Having in mind this interesting observation we decided to test the possibilities of direct inversion of GISAXS diffraction patterns in the range of exit angles from the specular $\left(\alpha_{f}=\alpha_{i}\right)$ to higher angles for different incidence conditions.

\section{Reconstruction of an island shape}

To invert coherent GISAXS diffraction patterns shown in Fig. 3 we used an iterative approach first proposed by Gerchberg and Saxton [37] and then further developed by Fienup [38]. What is important for this method of phase retrieval is the fact that no starting model is used for fitting the data. In the first step the missing phases are taken as a random set and are combined with the amplitudes in reciprocal space. Then FT is applied back and forth with specific constraints used in both real and reciprocal space. To test the reproducibility, the calculations are repeated with another set of starting random phases. To avoid stagnation we used an alternation of two algorithms: error-reduction (ER) (100 cycles) and hybrid inputoutput (HIO) [38] (50 cycles) having about 3000 cycles for each set of random phases. A detailed description of these algorithms and actual parameters used in reconstruction can be found in [15]. The following constraints were applied. In reciprocal space, the calculated amplitude on each iteration step was replaced by its experimentally measured amplitude $\sqrt{I_{\exp }(\mathbf{q})}$. In real space, using the fact that GISAXS measurements are nonsensitive to strain, we can take as a powerful constraint that the electron density has to be real and positive. An additional constraint tied to the concept of oversampling is that the density in real space is confined to a finite region called the support. We used the support region defined in the form of a rectangular window with the height (20 px) and width (17 px) that is slightly bigger than the height and width of our island.

Calculations of GISAXS diffraction patterns in Fig. 3 were performed for the momentum range $\Delta Q=0.08 \AA^{-1}$ in both horizontal and vertical directions. As soon as in the GISAXS 


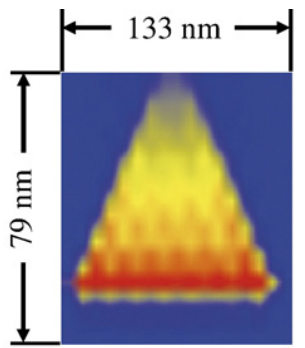

(a)

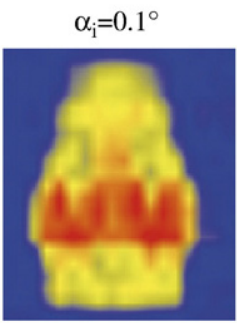

(b)

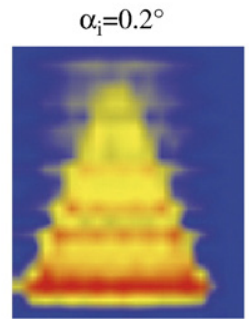

(c)

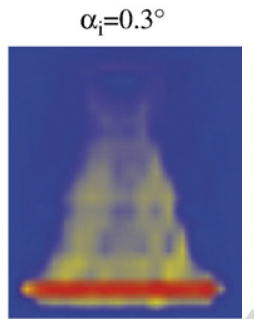

(d)

Fig. 4. Results of reconstruction of the individual island shape obtained from GISAXS diffraction patterns shown in Fig. 3. For comparison reconstruction of a single island from a kinematically scattered intensity (Fig. 3a) is also shown in (a). The total size of each image indicated in the figure correspond to the size of a support region used in calculations.

geometry measurements are not sensitive to strain the whole diffraction pattern containing both positive and negative $\mathbf{q}_{z}$ values should be centrosymmetric. Therefore, for the reconstruction of an island shape, the original GISAXS diffraction pattern was artificially symmetrized over its origin. After this operation we get from the initial size of the diffraction pattern $200 \times 200 \mathrm{px}^{2}$ the final one $400 \times 200 \mathrm{px}^{2}$ that was used in the reconstruction. Taking into account the size of the defined support region $20 \times 17 \mathrm{px}^{2}$ we see that diffraction patterns are highly oversampled. From the FFT relationship $\Delta x \Delta \mathbf{q}_{x}=$ $2 \pi / N_{x}$, where $N_{x}$ is a number of pixels in each direction, we have for the pixel size in real space: $\Delta y=78.5 \AA$ in horizontal direction and $\Delta z=39.25 \AA$ in the vertical. Taking into account that resolution is determined by the maximum momentum value we have $16 \mathrm{~nm}$ resolution in horizontal direction and $8 \mathrm{~nm}$ in the vertical.

Results of reconstruction of an island shape from GISAXS diffraction patterns for different incidence angles are presented in Fig. 4. For comparison, reconstruction of a particle shape solely from kinematically scattered intensity $I_{\text {kin }}(\mathbf{q})$ is also shown in Fig. 4a. From these figures we can distinctly see that for the incident angle of $0.2^{\circ}$ the shape of the island as well as its size is nearly perfectly reconstructed. However this is not the case for angles higher and lower than the critical angle. For angles smaller than the critical angle the shape of reconstructed island is corrupted by an overlapping image of a twin image (an island $180^{\circ}$ inverted to an original one). For angles larger than the critical angle no adequate reconstruction was obtained.

In order to analyze these results we performed calculations of each of the scattering channels depicted in Fig. 2 separately. Intensities $I_{i}\left(\mathbf{q}_{z}\right)=\left|A_{i}\left(\mathbf{q}_{z}\right)\right|^{2}, i=1, \ldots, 4$ corresponding to contribution of each scattering channel (Eqs. (4)-(7)) calculated at $\mathbf{q}_{y}=0$ for different incidence angles are shown in Fig. 5. As it follows from these calculations for incidence angle $\alpha_{i}=0.2^{\circ}$ and exit angle values higher than specular $\left(\alpha_{f}=\alpha_{i}\right)$ the dominating contribution to the total intensity is given by two scattering channels: the second one (wave specularly scattered from the surface and then kinematically scattered from the island (Fig. 2)) and kinematically scattered. Scattering from the 3-rd and the 4-th channels for these conditions is suppressed.

This situation can be also understood from the formal analysis of the expression for the total scattered amplitude $A_{\text {tot }}$ $\left(\mathbf{k}_{i}, \mathbf{k}_{f}\right)$ Eq. (3) including all four channels of scattering Eqs. (4)(7). The total amplitude can be written in a compact form as (compare similar expression obtained in [39])

$$
\begin{aligned}
A_{\text {tot }}\left(\mathbf{k}_{i}, \mathbf{k}_{f}\right)= & \chi_{0}\left(\frac{\mathbf{k}^{2}}{4 \pi}\right) \int s(\mathbf{r}) e^{-i \mathbf{q}_{\|} \boldsymbol{\rho}^{-i \mathbf{k}\left(\alpha_{i}+\alpha_{f}\right) z}} \\
& \times\left[1+R\left(\alpha_{i}\right) e^{2 i\left(\mathbf{k} \alpha_{i}\right) z}\right]\left[1+R\left(\alpha_{f}\right) e^{2 i\left(\mathbf{k} \alpha_{f}\right) z}\right] d \mathbf{r},
\end{aligned}
$$

where $\boldsymbol{\rho}$ is the component of vector $\mathbf{r}$ along the surface. For exit angles $\alpha_{f} \gg \alpha_{c}$ the reflectivity $\left|R\left(\alpha_{f}\right)\right| \ll 1$ and the last term in (a)

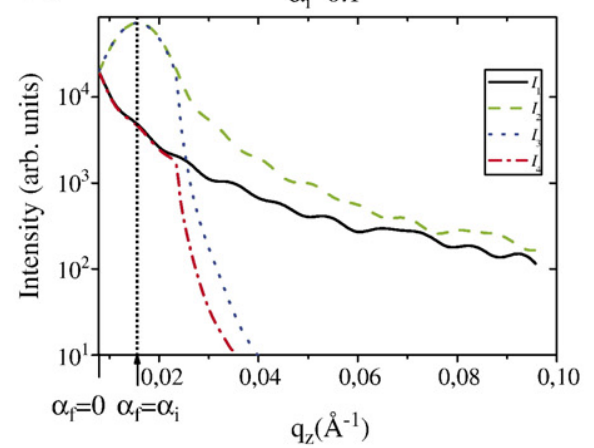

(b)

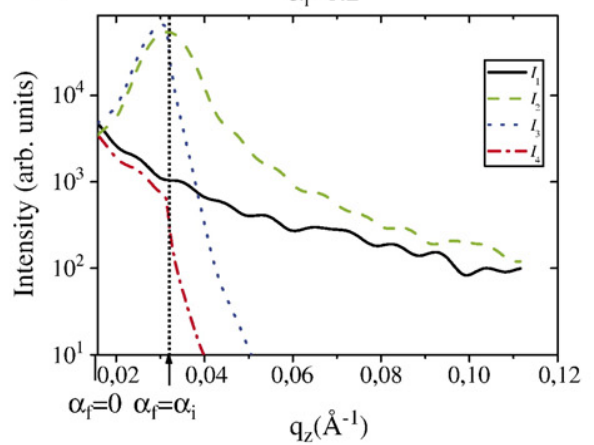

(c)

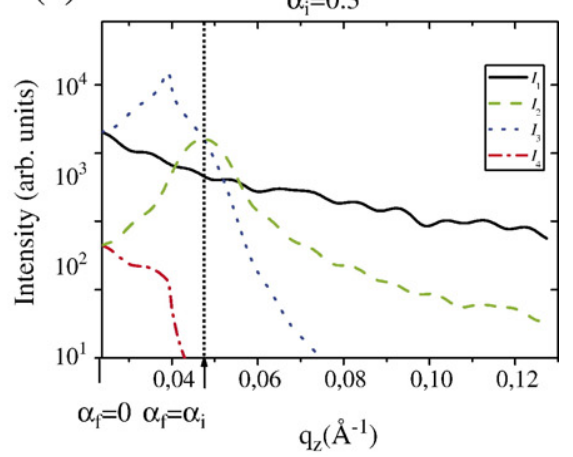

Fig. 5. Intensities $I_{i}\left(\mathbf{q}_{z}\right), i=1, \ldots, 4$ corresponding to contribution of each scattering channel (Eqs. (4)-(7)) calculated at $\mathbf{q}_{y}=0$ for different incidence angles. 
Eq. (9) can be neglected. For these scattering conditions the total amplitude $A_{\text {tot }}\left(\mathbf{k}_{i}, \mathbf{k}_{f}\right)$ reduces to

$$
\begin{aligned}
A_{\text {tot }}\left(\mathbf{k}_{i}, \mathbf{k}_{f}\right)= & \chi_{0}\left(\frac{\mathbf{k}^{2}}{4 \pi}\right) \int s(\mathbf{r})\left[R\left(\alpha_{i}\right)+e^{-2 i\left(\mathbf{k} \alpha_{i}\right) z}\right] \\
& \times e^{-i \mathbf{q}_{\|} \boldsymbol{\rho}-i \mathbf{k}\left(\alpha_{f}-\alpha_{i}\right) z} d \mathbf{r} .
\end{aligned}
$$

This expression in fact means that the total scattering including two channels can be described as originating from an island with the modified shape function that is now complex valued

$\tilde{s}(\mathbf{r})=s(\mathbf{r})\left[R\left(\alpha_{i}\right)+e^{-2 i\left(\mathbf{k} \alpha_{i}\right) z}\right]$.

Here we obtained an interesting result. The original shape of the particle can be described with a positive real valued function $s(\mathbf{r})$ that will produce a centrosymmetric diffraction pattern in the kinematical approximation. When rescattering effects from the flat surface are taken into account then the modified shape function $\tilde{s}(\mathbf{r})$ Eq. (11) becomes complex valued and will produce in general a non-centrosymmetric diffraction pattern. However, in the GISAXS geometry only the upper part of the reciprocal space $\mathbf{q}_{z}>0$ can be measured experimentally and for reconstruction the whole reciprocal space including $\mathbf{q}_{z}<0$ values have to be accessed. To go around that problem we artificially centrosymmetrized the diffraction pattern around its origin. From the properties of the Fourier transform [40] it is known that the amplitude of the Fourier transform of a real positive function will be centrosymmetric. So, to be consistent, we put that constrain (real and positive object) in the real space. It means that in the reconstruction we can obtain only a modulus of the real part of the modified shape function in Eq. (11)

$$
|\operatorname{Re}[\tilde{s}(\mathbf{r})]|=s(\mathbf{r})\left|\left[\left|R\left(\alpha_{i}\right)\right| \cos \varphi\left(\alpha_{i}\right)+\cos \left(2 \mathbf{k} \alpha_{i} z\right)\right]\right|
$$

where $\varphi\left(\alpha_{i}\right)$ is the phase of the complex reflectivity $R\left(\alpha_{i}\right)$. For incident angles $\alpha_{i}=\alpha_{c}$ with a good approximation $\left|R\left(\alpha_{i}\right)\right| \simeq 1$ and $\varphi\left(\alpha_{i}\right) \simeq 0$, we get from Eq. (12)

$|\operatorname{Re}[\tilde{s}(\mathbf{r})]|=s(\mathbf{r})\left[1+\cos \left(2 \mathbf{k} \alpha_{i} z\right)\right]$

According to this expression the original shape function $s(\mathbf{r})$ is modulated by periodic function with the period in the vertical direction $\Delta z=\pi /\left(\mathbf{k} \alpha_{i}\right)$. For the incidence angle of $0.2^{\circ}$ and our incoming energies we get for that period $\Delta z=20 \mathrm{~nm}$. That gives an explanation for the vertical bands that appear as an artifact in the reconstructed image in Fig. 4c.

The situation is quite different for incidence angles smaller and higher than the critical angle. At $\alpha_{i}<\alpha_{c}$ (Fig. 5a) contributions of the second channel and the kinematically scattered channel are starting to be comparable in their magnitude. In addition, at these incidence angles the reflectivity amplitude $R\left(\alpha_{i}\right)$ has a magnitude of one and is rapidly changing its phase from zero to $-\pi$. Contributions of channels three and four are comparable to that of channels one and two (see Fig. 5a) as soon as an absolute value of the reflectivity $\left|R\left(\alpha_{f}\right)\right|$ is about one for that angular range. However this is valid only for very limited values of exit angles $\alpha_{i}<\alpha_{f}<\alpha_{c}$. Finally, considering contribution of two channels to the total amplitude $A_{\text {tot }}\left(\mathbf{k}_{i}\right.$, $\mathbf{k}_{f}$ ) for these scattering conditions gives for the modified shape function $\tilde{s}(\mathbf{r})$ in Eq. (12)

$|\operatorname{Re}[\tilde{s}(\mathbf{r})]|=s(\mathbf{r})\left|\left[1-\cos \left(2 \mathbf{k} \alpha_{i} z\right)\right]\right|=2 s(\mathbf{r}) \sin ^{2}\left(\mathbf{k} \alpha_{i} z\right)$.

In the limit of very small incidence angles $\alpha_{i}$ this expression gives

$|\operatorname{Re}[\tilde{s}(\mathbf{r})]|=2\left(\mathbf{k} \alpha_{i} z\right)^{2} s(\mathbf{r})$.

According to Eq. (15) at very small incidence angles $\alpha_{i}$, the shape function $s(\mathbf{r})$ is modified by the quadratic function $z^{2}$. This effect can be observed in the reconstructed image in Fig. $4 \mathrm{~b}$. It reveals itself as a broad maximum in the center of the image.

For incidence angles higher than the critical $\alpha_{i}>\alpha_{c}$ (Fig. 5c) the strongest channel of scattering is the kinematical one. Both reflectivity amplitudes $\left|R\left(\alpha_{i}\right)\right| \ll 1$ and $\left|R\left(\alpha_{f}\right)\right| \ll 1$ for these scattering conditions and the expression for the total amplitude $A_{\text {tot }}\left(\mathbf{k}_{i}, \mathbf{k}_{f}\right)$ Eq. (9) reduces to

$A_{\text {tot }}\left(\mathbf{k}_{i}, \mathbf{k}_{f}\right)=\chi_{0}\left(\frac{\mathbf{k}^{2}}{4 \pi}\right) \int s(\mathbf{r}) e^{-2 i\left(\mathbf{k} \alpha_{i}\right) z} e^{-i \mathbf{q}_{\|} \boldsymbol{\rho}^{-i \mathbf{k}\left(\alpha_{f}-\alpha_{i}\right) z}} d \mathbf{r}$.

This gives for the modified shape function $\tilde{s}(\mathbf{r})$ that is actually obtained in reconstruction procedure

$|\operatorname{Re}[\tilde{s}(\mathbf{r})]|=\left|\operatorname{Re}\left[s(\mathbf{r}) e^{-2 i\left(\mathbf{k} \alpha_{i}\right) z}\right]\right|=s(\mathbf{r})\left|\cos \left(2 \mathbf{k} \alpha_{i} z\right)\right|$.

We see that the original shape function $s(\mathbf{r})$ is modified with the fast oscillating periodic function with the period $\Delta z \sim 8 \mathrm{~nm}$ for our conditions. That is about one pixel size in the vertical direction in Fig. 4d and certainly cannot be resolved in our case. That analysis explains why reconstruction made from the GISAXS diffraction pattern at angles $\alpha_{i}>\alpha_{f}$ fails to give a correct shape of an island as shown in Fig. $4 \mathrm{~d}$.

\section{Conclusions}

In this paper we analyzed coherent scattering from an island of nanometer size in GISAXS scattering geometry at small incidence angles below and above the critical angle. With these scattering conditions the process cannot be described as purely kinematical and three other scattering channels have to be taken into account. All four scattering channels contribute coherently to the total amplitude. Experimentally measured GISAXS intensity is a sum of intensities corresponding to each scattering channel plus interference terms between each of them. In this scattering geometry, application of the phase retrieval methods based on application of Fourier transform back and forth between real and reciprocal space looks not feasible. However, our analysis has shown that there are some favorable conditions with incident angle close to the critical angle when GISAXS diffraction pattern originating from the individual island of nano-size dimensions can be directly inverted using iterative phase retrieval technique and can give a reliable image of an 
island shape. In our future work we plan to take into account in the iteration procedure effects produced by modified shape function as described in this paper. This could produce even better results free from artifacts discussed in the previous section.

We think that our results on model independent reconstruction of an island shape from the coherent GISAXS measurements can lead to a development of a new and powerful technique for investigating the structure of nanosamples.

\section{Acknowledgment}

The authors are thankful to R. Köhler, J. Schneider and E. Weckert for their permanent interest and support of this work. They are also thankful to S. Boutet for a careful reading of the manuscript. The research of D. Grigoriev was also partially supported by SFB 296 Project.

\section{References}

[1] R. Bates, Optik 61 (1982) 247.

[2] J.R. Fienup, Opt. Lett. 3 (1978) 27.

[3] D. Sayre, in: M. Schlenker (Ed.), Imaging Processes and Coherence in Physics, Springer-Verlag, Berlin, 1980.

[4] I.A. Vartanyants, J.A. Pitney, J.L. Libbert, I.K. Robinson, Phys. Rev., B 55 (1997) 13193

[5] J. Miao, P. Charalambous, J. Kirz, D. Sayre, Nature 400 (1999) 342.

[6] I.K. Robinson, J.L. Libbert, I.A. Vartanyants, J.A. Pitney, D.M. Smilgies, D.L. Abernathy, G. Grübel, Phys. Rev., B 60 (1999) 9965.

[7] S. Marchesini, H.N. Chapman, S.P. Hau-Riege, R.A. London, A. Szoke, H. He, M.R. Howells, H. Padmore, R. Rosen, J.C.H. Spence, U. Weierstall, Opt. Express 11 (2003) 2344.

[8] I.K. Robinson, I.A. Vartanyants, G.J. Williams, M.A. Pfeifer, J.A. Pitney, Phys. Rev. Lett. 87 (2001) 195505.

[9] J. Miao, T. Ishikawa, B. Johnson, E. Andersen, B. Lai, K. Hodgson, Phys. Rev. Lett. 89 (2002) 088303.

[10] H.N. Chapman, A. Barty, S. Marchesini, A. Noy, C. Cui, M.R. Howells, R. Rosen, H. He, J.C.H. Spence, U. Weierstall, T. Beetz, C. Jacobsen, D. Shapiro, J. Opt. Soc. Am. 23 (2006) 1179.

[11] A. Schropp, I. Vartanyants, C.G. Schroer, E. Weckert, C. Mocuta, T. Metzger, O. Kuparova, Proc. 8th Int. Conf. X-ray Microscopy, IPAP Conf Series, vol. 7, 2006, p. 383

[12] D. Shapiro, P. Thibault, T. Beetz, V. Elser, M. Howells, C. Jacobsen, J. Kirz, E. Lima, H. Miao, A.M. Neiman, D. Sayre, PNAS 102 (2005) 15343.

[13] G.J. Williams, M.A. Pfeifer, I.A. Vartanyants, I.K. Robinson, Phys. Rev. Lett. 90 (2003) 175501.
[14] G.J. Williams, M.A. Pfeifer, I.A. Vartanyants, I.K. Robinson, Phys. Rev., B 73 (2006) 094112.

[15] I.A. Vartanyants, I.K. Robinson, J. Phys., Condens. Matter 13 (2001) 10593.

[16] M.A. Pfeifer, G.J. Williams, I.A. Vartanyants, R. Harder, I.K. Robinson, Nature 442 (2006) 63.

[17] M. Altarelli, et al., (Eds.), XFEL The European X-ray Free-Electron Laser, Technical Design Report, 2006, (DESY 2006-097) http://xfel.desy.de/tdr/ index_eng.html.

[18] J. Arthur, et al., Linac Coherent Light Source (LCLS) Conceptual Design Report. http://www-ssrl.slac.stanford.edu/LCLS/CDR/.

[19] R. Neutze, R. Wouts, D. Spoel, E. Weckert, J. Hajdu, Nature 406 (2000) 752.

[20] D. Bimberg, M. Grundmann, N.N. Ledentsov, Quantum Dot Heterostructures, Wiley, Chichester, 1999.

[21] U. Pietsch, V. Holy, T. Baumbach, High-resolution X-ray scattering from thin films to lateral nanostructures, Advanced Texts in Physics. 2nd ed., Springer, Berlin, 2004.

[22] M. Schmidbauer, X-ray Diffuse Scattering from Self-Organized Mezoscopic Semiconductor Structures, Springer, Berlin, 2004.

[23] I.A. Vartanyants, I.K. Robinson, J. Synchrotron Radiat. 10 (2003) 409.

[24] I.A. Vartanyants, I.K. Robinson, J.D. Onken, M.A. Pfeifer, G.J. Williams, F. Pfeiffer, H. Metzger, Z. Zhong, G. Bauer, Phys. Rev., B 71 (2005) 245302.

[25] I.K. Robinson, F. Pfeiffer, I.A. Vartanyants, Y. Sun, Y. Xia, Opt. Express 11 (2003) 2329.

[26] C.G. Schroer, O. Kurapova, J. Patommel, et al., Appl. Phys. Lett. 87 (2005) 124103.

[27] H.C. Kang, J. Maser, G.B. Stephenson, et al., Phys. Rev. Lett. 96 (2006) 127401.

[28] J. Stangl, G. Bauer, I.A. Vartanyants, C. Mocuta, H.T. Metzger, in press.

[29] J. Als-Nielsen, D. McMorrow, Elements of Modern X-ray Physics, John Wiley \& Sons, New York, 2001.

[30] S.K. Sinha, E.B. Sirota, S. Garoff, H.B. Stanley, Phys. Rev., B 38 (1988) 2297.

[31] M. Rauscher, R. Paniago, H. Metzger, et al., J. Appl. Phys. 86 (1999) 6763.

[32] R. Lazzari, J. Appl. Crystallogr. 35 (2002) 406.

[33] L.D. Landau, E.M. Lifshits, Quantum Mechanics-Nonrelativistic Theory, 2nd ed.Pergamon Press, Oxford, 1965.

[34] M. Born, E. Wolf, Principles of Optics, Electromagnetic Theory of Propagation, Interference and Diffraction of Light, Chapter XIII, 7th ed., University Press, Cambridge, 1999.

[35] M. Schmidbauer, D. Grigoriev, M. Hanke, et al., Phys. Rev., B 71 (2005) 115324 .

[36] I.K. Robinson, Phys. Rev., B 33 (1986) 3830.

[37] R.W. Gerchberg, W.O. Saxton, Optik 35 (1972) 237.

[38] J.R. Fienup, Appl. Opt. 21 (1982) 2758.

[39] I. Kegel, T.H. Metzger, A. Lorke, et al., Phys. Rev., B 63 (2001) 035318

[40] See for e.g. W.H. Press, S.A. Teukolsky, W.T. Vetterling, B.P. Flannery, Numerical Recipes in Fortran 77, second edition, The Art of Scientific Computing, vol. 1, Cambridge University Press, 1996, p. 490. 